\title{
Quantitative Prediction of the Phase Diagram of DNA-Functionalized Nanosized Colloids
}

\author{
Bianca M. Mladek, ${ }^{1,2, *}$ Julia Fornleitner, ${ }^{3}$ Francisco J. Martinez-Veracoechea, ${ }^{1}$ Alexandre Dawid, ${ }^{4}$ and Daan Frenkel ${ }^{1}$ \\ ${ }^{1}$ Department of Chemistry, University of Cambridge, Lensfield Road, Cambridge, CB2 1EW, United Kingdom \\ ${ }^{2}$ Department of Structural and Computational Biology, Max F. Perutz Laboratories GmbH, University of Vienna, 1030 Vienna, Austria \\ ${ }^{3}$ Institute for Complex Systems, Forschungszentrum Jülich, 52428 Jülich, Germany \\ ${ }^{4}$ Laboratoire Interdisciplinaire de Physique UMR 5588, Université Joseph Fourier Grenoble 1/CNRS, Grenoble, 38041, France
}

(Received 19 March 2012; published 25 June 2012)

\begin{abstract}
We present a coarse-grained model of DNA-functionalized colloids that is computationally tractable. Importantly, the model parameters are solely based on experimental data. Using this highly simplified model, we can predict the phase behavior of DNA-functionalized nanocolloids without assuming pairwise additivity of the intercolloidal interactions. Our simulations show that, for nanocolloids, the assumption of pairwise additivity leads to substantial errors in the estimate of the free energy of the crystal phase. We compare our results with available experimental data and find that the simulations predict the correct structure of the solid phase and yield a very good estimate of the melting temperature. Current experimental estimates for the contour length and persistence length of single-stranded (ss) DNA sequences are subject to relatively large uncertainties. Using the best available estimates, we obtain predictions for the crystal lattice constants that are off by a few percent: this indicates that more accurate experimental data on ssDNA are needed to exploit the full power of our coarse-grained approach.
\end{abstract}

DOI: 10.1103/PhysRevLett.108.268301

PACS numbers: 82.70.Dd, 07.05.Tp, 64.70.Nd, 64.75.Yz

Nature provides spectacular examples of complex, functional systems that self-assemble from small, prefabricated units. The grand challenge in nanomaterial design is to imitate this phenomenon to construct complex, functional materials. To achieve this goal, we must be able to design suitable nanosized building blocks and to create conditions that cause these entities to self-assemble into the desired target structures. DNA-based building blocks-which take advantage of the selective binding of bases on complementary strands to guide assemblyoffer attractive model systems to explore self-assembly strategies (see, e.g., Refs. [1,2]). For instance, colloidal particles can be functionalized with a short singlestranded (ss) DNA sequence tethered to an inert polymeric "spacer" [3]. These "sticky ends" on two different colloids may then either bind directly to each other via complementary sequences or via a ssDNA linker sequence introduced in a solution, allowing the colloids to form three-dimensional structures. The properties of these building blocks, temperature, $p \mathrm{H}$, and ionic strength of the parent solution determine if, and on what time scale, self-assembly takes place.

In view of the vastness of this "design space" [4], a careful selection of experimental conditions is crucial: experiments on nano- [5-10] and micronsized DNAfunctionalized colloids (DNACs) [11,12] show that the self-assembly of spatially ordered structures requires considerable fine tuning: under most experimental conditions, amorphous aggregates form, even if the ordered (crystal) phase is thermodynamically stable. The experiments show that the stability of crystals depends crucially on temperature $(T)$ and on the length and flexibility of the spacers. Of the crystal structures experimentally observed, the bcc and fcc lattices are the most stable, while to date little progress has been made with the self-assembly of DNAC crystals that are more complex than cubic. Therefore, it is desirable to have theoretical and numerical guidance in selecting optimal conditions for self-assembly. However, an ab initio approach to studying the phase behavior of DNACs quantitatively, using many-particle simulations, goes well beyond the state of the art. Existing theoretical studies are based on the assumption that the interaction between DNACs is pairwise additive [13], and numerical simulations of DNACs typically employ highly simplified, ad hoc coarse-grained models that also assume pairwise additivity of interactions (see, e.g., Refs. [14-22]). An exception is the work of Rogers et al. [23,24]. However, the approach followed in their paper, while suited to computing pair potentials between micronsized DNACs, would become computationally prohibitively expensive for nanosized DNACs, where, as we will show below, it is not warranted to assume pairwise additivity of the intercolloidal interactions. What is more, those systems that have been shown most promising for crystallization are nano-DNACs covered with DNA strands in an intermediate length regime, where the DNA strands are too long to be approximated within the rod picture [20] but too short to make use of polymer scaling laws. As a consequence, existing models cannot be used to predict the stability of crystal phases of nano-DNACs.

In this Letter, we present a quantitative numerical approach to predict the thermodynamic stability and phase behavior of nano-DNACs based on a coarse-graining procedure free of fitting parameters. 
We validate our coarse-graining approach on a system that has been studied extensively in experiments [6], namely, a symmetric, binary mixture of gold nanocolloids of a radius $R_{C} \sim 6 \mathrm{~nm}$ which were grafted with $\sim 60$ ssDNA strands. Colloidal species $A$ and $B$ only differ in the sequence of their sticky ends, which mediate the binding between colloids $A$ and $B$. In the experiments of Ref. [6], it was observed that systems with ssDNA strands of more than 50 nucleotides crystallize into $\mathrm{CsCl}$ structures. In our Letter, we therefore focus on their system of DNACs functionalized with ssDNA strands of 65 nucleotides, 15 of which were responsible for binding. These DNACs were experimentally found to crystallize for temperatures below $T_{m}^{\exp }=62.5^{\circ} \mathrm{C}$.

In order to arrive at a computationally tractable model, we carry out a staged coarse-graining procedure. At the most microscopic (yet not fully atomistic) level, we represent the Au colloid as a hard sphere of radius $R_{C}$ and we model the ssDNA strands as freely jointed, charged chains, with a Kuhn length of $1.5 \mathrm{~nm}[25,26]$, and using an interbase distance for ssDNA of $0.43 \mathrm{~nm}$ [26]. We stress, however, that the values reported in these (and other) papers are subject to considerable error bars and are likely to be sensitive to both the physical conditions of the solution and the precise DNA sequence. Hence, we should expect that these inaccuracies will translate into errors in computed characteristic length scales, such as the lattice constants of DNAC crystals. As we show below, this is precisely what we observe.

The charge carried by the ssDNA's backbone is assumed to be distributed equally among the vertices of the freely jointed chain. The vertices interact through a DebyeHückel potential that depends on $T$ and on the dielectric constant and ionic strength of the solvent [25]. We assume that the grafted DNA is distributed uniformly on the Au surface and does not diffuse [27].

In spite of the simplicity of this model, it has a large number of degrees of freedom per colloid $(\sim 3000)$. As a consequence, simulations are only feasible for relatively small systems. In order to be able to treat DNA hybridization in systems containing many colloids, we therefore develop the next level of coarse graining: a "core-blob" model in which each sticky end is coarse-grained to a polymer "blob," while the bare colloid and the remaining segments of all strands constitute an effective colloidal "core." In this way, we reduce the degrees of freedom per colloid tenfold. We determine the parameters and interaction potentials that characterize the core-blob model in Monte Carlo simulations of the underlying microscopic model [28]. Further, we allow for DNA hybridization in the core-blob model. The binding probability of complementary DNA strands is computed on the basis of the tabulated hybridization free energy of two complementary ssDNAs in solution [29], which is directly based on experimental data. Details of the coarse-graining procedure and the
Monte Carlo scheme used to carry hybridization moves will be presented elsewhere [30].

In what follows, we will use the core-blob model to predict the phase behavior of DNACs. However, we first go one step further in coarse graining and compute the effective pair potentials $\Phi_{2}^{\times}(r, T)$ between two DNACs $(\boldsymbol{\aleph}=\{A A, B B, A B\})$ as a function of the distance $r$ and of $T$. $\Phi_{2}^{A A, B B}(r, T)$ accounts for the steric repulsion $\Phi_{2 \text {,rep }}$ between the colloids, which can be determined as detailed in [28]. In the $A B$ case, there is an additional attractive hybridization interaction $\Phi_{2 \text {,hyb }}$, which is proportional to the amount of hybridized DNA strands and is calculated by thermodynamic integration, as is described in Ref. [20]. We find that $\Phi_{2 \text {,rep }}$ is nearly constant between 25 and $75^{\circ} \mathrm{C}$, while $\Phi_{2, \text { hyb }}$ is strongly $T$-dependent (Fig. 1). For $T \lesssim T_{m}^{\exp }$, $\Phi_{2}^{A B}$ therefore develops a strongly $T$-dependent minimum at distances $r \sim 5.25$ to $6 R_{C}$. For the system presented here, a $T$ difference of only $5.2{ }^{\circ} \mathrm{C}\left(62.1\right.$ to $\left.56.9^{\circ} \mathrm{C}\right)$ leads to a difference in minimum in $\Phi_{2}^{A B}$ of $\sim 20 k_{B} T \sim 13 \mathrm{kcal} / \mathrm{mol}$. The difficulty of making high-quality crystals of DNACs is related to this strong temperature dependence of $\Phi_{2}^{A B}$ : defects in growing crystals can only anneal if $T$ is just below the melting temperature. At lower $T$, bonds are very stable and dense aggregates, once formed, cannot equilibrate. We are unaware of direct measurements of the pair potentials of DNA-functionalized nanocolloids; hence, we cannot vali-

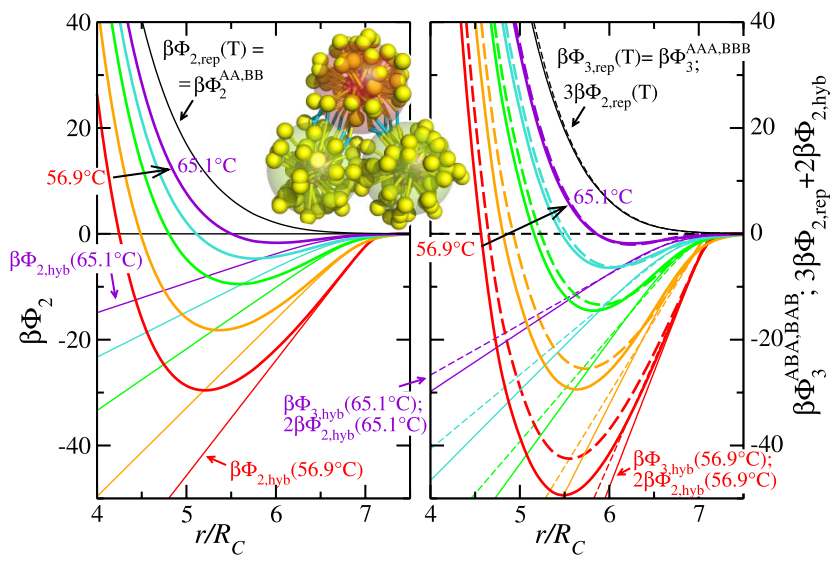

FIG. 1 (color online). Left: The pair interaction $\Phi_{2}^{A B}(r, T)$ (bold lines) is the sum of the $T$-independent steric repulsion $\Phi_{2 \text {,rep }}(r)$ and the strongly $T$-dependent attractive hybridization energy $\Phi_{2, \text { hyb }}(r, T)$ (both as labeled). Right: The three-body interaction $\beta \Phi_{3}^{A B A}(r)$ (bold dashed lines) of two $A$ and one $B$ colloids arranged in an equilateral triangle (inset) compared with the sum of the two-body contributions $3 \beta \Phi_{2 \text {,rep }}+2 \beta \Phi_{2 \text {,hyb }}$ (bold solid lines). The repulsive and the attractive hybridization contributions to the interactions are shown explicitly in thin lines (two-body, dashed lines; three-body, solid lines). Both: Results are shown at $56.9^{\circ} \mathrm{C}, 59.1^{\circ} \mathrm{C}, 61.4^{\circ} \mathrm{C}, 63.2^{\circ} \mathrm{C}$, and $65.1^{\circ} \mathrm{C}$. Inset: A simulation snapshot of one $B$ colloid (top) interacting with two $A$ (bottom) at $59.1{ }^{\circ} \mathrm{C}$ and $r=5.75 R_{C}$. Free blobs are drawn as small spheres, hybridized sticky ends as rods. Translucent spheres indicate the extension of the core. 
date our model at this level. However, numerically, we can test whether the assumption of pairwise additivity of $\Phi_{2}^{\mathrm{N}}$, as typically employed in theoretical studies, is warranted. To this end, we compute the interaction $\Phi_{3}^{\aleph}(r, T) \quad(\aleph=$ $\{A A A, B B B, A B A, B A B\}$ ) between a triplet of colloids arranged on the vertices of an equilateral triangle with side length $r$ (inset of Fig. 1). We find that $\Phi_{3}^{A A A, B B B}=\Phi_{3 \text {,rep }}$ is well represented by $3 \Phi_{2 \text {,rep }}$ for all $T$ considered. For the $A B A(=B A B)$ case, additivity would imply $\Phi_{3}^{A B A}(r, T)=$ $3 \Phi_{2 \text {,rep }}(r, T)+2 \Phi_{2, \text { hyb }}(r, T)$. As can be seen in the right panel of Fig. 1, even for temperatures close to $T_{m}^{\exp }$, this relation is only fulfilled at large separations $r \geqslant 6 R_{C}$. The reason why additivity fails in this case is that different $B$ colloids compete for the same DNA on $A$. This effect is not accounted for in $\Phi_{2}$, which measures the average amount of bonds in an isolated $A B$ pair and hence overestimates the attraction between $A B$ pairs in a trimer [31].

Turning our attention to multiparticle systems, we study the thermodynamic stability of various crystalline phases. Using the pair potentials, we first identify candidate equilibrium crystal structures via a genetic algorithm similar to the one used in Refs. [32,33]. This approach, which neglects positional entropy, suggests that the $\mathrm{CsCl}$ structure is most stable close to the melting density, while a NaTl structure is predicted to be stable at higher densities. Experimentally, the distinction between these two structures is not straightforward, as x-ray scattering only probes the arrangement of the Au cores, which is the same for $\mathrm{CsCl}$ and $\mathrm{NaTl}$ crystals. We also considered $\mathrm{CuAu}, \mathrm{NaCl}$, "straight" hep (s-hep), ZnS, AuCd, and substitutionally disordered $\mathrm{CsCl}$ and $\mathrm{CuAu}$ structures. For all crystal structures and also the fluid phase, we compute the free energy $F$ for $T \lesssim T_{m}^{\exp }$ via thermodynamic integration within the core-blob model (Fig. 2). The $\mathrm{CsCl}$ phase is found to have the lowest free energy for low colloidal volume fractions $\eta$. Only slightly higher in $F$, we find metastable s-hcp, $\mathrm{CuAu}$, and $\mathrm{NaTl}$ structures. While the $\mathrm{CsCl}$ is already mechanically stable for $\eta=0.023$, the latter structures are only mechanically stable for slightly higher $\eta \gtrsim$ 0.026-0.035. At high $\eta \sim 0.065$, a metastable AuCd phase appears. The $\mathrm{NaCl}$ and $\mathrm{ZnS}$ structures are mechanically unstable for all $\eta$ considered. For $\eta \geqslant 0.07$, the NaTl structure (composed of two interpenetrating diamond structures) competes-as predicted-with the $\mathrm{CsCl}$ structure; however, this is in a density regime where the validity of our model is not guaranteed. Moreover, such dense crystals cannot easily be prepared in experiments.

We stress that the formation of the low-density crystals is due to the DNA links between colloids. At much higher $\eta$, the crystal structure will be dictated by excluded volume interactions rather than DNA links. Then, close-packed structures such as $\mathrm{CuAu}$ and s-hcp should be more stable than the more open structures that dominate at lower $\eta$. We also consider substitutional disorder and find that strongly disordered crystal structures are only mechanically stable

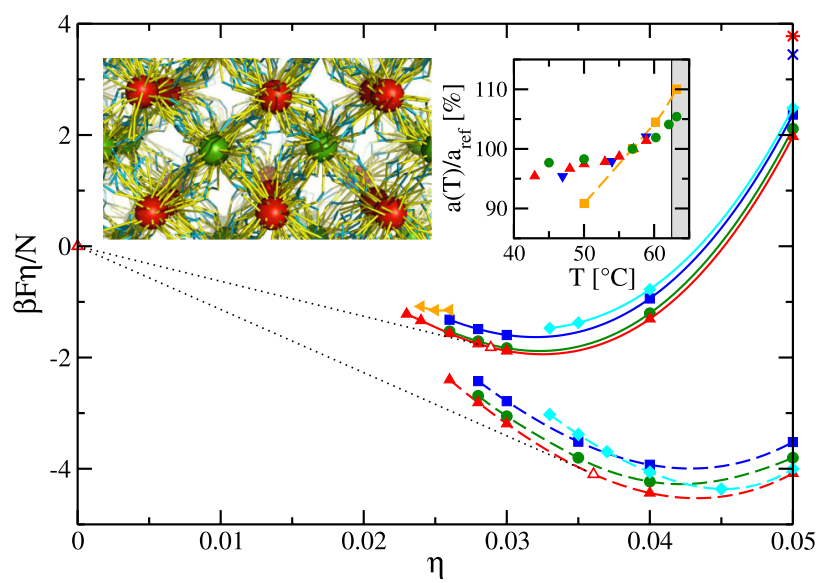

FIG. 2 (color online). The dimensionless free energy $\beta \eta F / N$ at $T=56.9^{\circ} \mathrm{C}$ as function of the colloidal volume fraction $\eta$ shows that the $\mathrm{CsCl}$ structure is the most stable one. Pair potential approach, dashed lines; core-blob approach, solid lines. $\mathrm{CsCl}, \mathbf{\Delta}$; s-hcp, $\mathbf{O} \mathrm{CuAu}, \mathbf{\square} ; \mathrm{NaTl}, \diamond$; disordered $\mathrm{CsCl}$, *; disordered $\mathrm{CuAu}$, $\times$; and liquid, 4 . The common tangents between the equilibrium $\mathrm{CsCl}$ crystal and the dilute vapor are shown as dotted lines for both models. Right inset: the lattice constant $a$ as function of $T$ in ${ }^{\circ} \mathrm{C}$ as measured in the experiments (heating, $\mathbf{\Delta}$; cooling, $\boldsymbol{\nabla}$; data from Ref. [6]) and as obtained from simulations with the core-blob model (O) and using the pair potential approach (ם). The data of $a$ have been scaled to the values of $a_{\text {ref }}=a\left(56.9^{\circ} \mathrm{C}\right)$, as measured with the respective approaches: $a_{\mathrm{ref}}^{\exp }=45.3 \mathrm{~nm}, a_{\mathrm{ref}}^{\text {core-blob }}=39.7 \mathrm{~nm}$, and $a_{\text {ref }}^{\Phi_{2}}=36.9 \mathrm{~nm}$. In the shaded region, crystals are not stable in experiments. Left inset: a cut through a simulated $\mathrm{CsCl}$ structure at $\eta=0.024$ and $T=56.9^{\circ} \mathrm{C}$. For a better visualization, only the hybridized links are drawn.

for $\eta \gtrsim 0.05$ and less stable than the corresponding ordered structures. However, some substitutional disorder is inevitable: at $56.9^{\circ} \mathrm{C}$, the free-energy cost of a single $A B$ exchange in an otherwise ordered crystal structure ranges from $\Delta F=0.5 k_{B} T(\eta=0.024)$ to $1.6 k_{B} T(\eta=0.05)$ for $\mathrm{CsCl}$ and from $\Delta F=0.1 k_{B} T(\eta=0.026)$ to $1.0 k_{B} T$ ( $\eta=$ 0.05 ) for $\mathrm{CuAu}$. We note that substitutionally disordered crystals have been observed both in experiments [5] and in simulations [22]. The present results suggest that slightly disordered structures are metastable but kinetically arrested for $T \lesssim T_{m}^{\exp }$, while strongly disordered crystals will melt at low $\eta$. In Fig. 2, we further compare $F$ as obtained from the core-blob approach with the corresponding quantity obtained employing the pair potentials. The assumption of pairwise additivity of $\Phi_{2}$ leads to a serious underestimate of $F$. Nevertheless, for $T \leqq T_{m}^{\exp }$, this approach provides a reasonable estimate of the range of mechanical stability of the various crystal structures and predicts the same phase order as the core-blob model, albeit in a more compressed $\eta$ range.

We also used the core-blob model to test whether the colloidal suspension could undergo a transition between a dilute and a concentrated unordered phase below the freezing density and for $T \lesssim T_{m}^{\exp }$. We find that this is 
not the case: the $\mathrm{CsCl}$ structure directly coexists with a very dilute suspension [34]. Using the common-tangent construction (see Fig. 2), we can determine the volume fraction-and thereby the lattice constant $a$ - of the $\mathrm{CsCl}$ structure at coexistence. A comparison of the experimentally determined $a$ at various $T$ with the values obtained from the core-blob approach (right inset of Fig. 2) shows that the latter approach can predict the correct temperature dependence of the lattice spacing. This is not the case for the pair potential approach, which predicts an excessive contraction of the crystals. Both computational approaches predict lower values of $a$ than observed experimentally. The core-blob approach underestimates $a$ by $\sim 12 \%$, a discrepancy to be expected in view of the incomplete experimental information on the contour length and persistence length of ssDNA. With better experimental data, we expect that the core-blob model will yield a quantitative prediction of the DNAC crystal lattice constants. The predictions of the lattice constants as obtained by the use of pair potentials are qualitatively wrong-in particular, this approach incorrectly predicts the temperature dependence of the lattice spacing. We can further compare the experimentally determined crystal melting point with the melting point of simulated crystal slabs in equilibrium with the dilute vapor. In these simulations, we find that crystals melt for $T \geqslant 64.3(5){ }^{\circ} \mathrm{C}$, which matches well with the experimentally determined melting temperature $T_{m}^{\exp }=$ $62.5(3){ }^{\circ} \mathrm{C}$ for the 65 nucleotide system of Ref. [6]. The good quantitative agreement between simulation and experiment in melting temperature is significant, as the coreblob model contains no a posteriori adjustable parameters.

The present approach has been developed to model DNACs with direct hybridization between sticky ends. However, our approach can be generalized to describe binding via linker sequences and it can be adapted to describe asymmetric and polydisperse systems, to name but a few examples. Although some adjustment of the underlying microscopic model of DNACs is needed to mend the accuracy of length predictions, our approach offers a path to the computer-aided design of DNAfunctionalized building blocks that could be used to construct truly complex self-assembling structures.

We thank B. Capone, S. Angioletti-Uberti, B. M. Mognetti, W. Jacobs, and G. Day for useful discussions. This work was supported by funding from the EU (IEF2008 No. 236663, CIG-2011 No. 303860), the MFPL VIPS program, EMBO, ERC (Advanced Grant No. 227758), the Royal Society, and the EPSRC (No. EP/I001352/1).

*bianca.mladek@univie.ac.at

[1] R. Bashir, Superlattices Microstruct. 29, 1 (2001).

[2] F. A. Aldaye, A. L. Palmer, and H. F. Sleiman, Science 321, 1795 (2008)
[3] C.A. Mirkin, R.L. Letsinger, R.C. Mucic, and J.J. Storhoff, Nature (London) 382, 607 (1996).

[4] N. Geerts and E. Eiser, Soft Matter 6, 4647 (2010).

[5] S. Y. Park, A. K. R. Lytton-Jean, B. Lee, S. Weigand, G. C. Schatz, and C.A. Mirkin, Nature (London) 451, 553 (2008).

[6] D. Nykypanchuk, M. M. Maye, D. van der Lelie, and O. Gang, Nature (London) 451, 549 (2008).

[7] R. J. Macfarlane, B. Lee, H. D. Hill, A. J. Senesi, S. Seifert, and C.A. Mirkin, Proc. Natl. Acad. Sci. U.S.A. 106, 10493 (2009).

[8] H. Xiong, D. van der Lelie, and O. Gang, Phys. Rev. Lett. 102, 015504 (2009).

[9] R. J. Macfarlane, M. R. Jones, A. J. Senesi, K. L. Young, B. Lee, J. Wu, and C. A. Mirkin, Angew. Chem., Int. Ed. Engl. 49, 4589 (2010).

[10] P. Cigler, A. K. R. Lytton-Jean, D. G. Anderson, M. G. Finn, and S. Y. Park, Nature Mater. 9, 918 (2010).

[11] P. L. Biancaniello, A. J. Kim, and J. C. Crocker, Phys. Rev. Lett. 94, 058302 (2005).

[12] A. J. Kim, P. L. Biancaniello, and J. C. Crocker, Langmuir 22, 1991 (2006).

[13] A. V. Tkachenko, Phys. Rev. Lett. 89, 148303 (2002).

[14] D. B. Lukatsky and D. Frenkel, Phys. Rev. Lett. 92, 068302 (2004).

[15] F. Pierce, C. M. Sorensen, and A. Chakrabarti, Langmuir 21, 8992 (2005).

[16] F. W. Starr and F. Sciortino, J. Phys. Condens. Matter 18, L347 (2006).

[17] B. Bozorgui and D. Frenkel, Phys. Rev. Lett. 101, 045701 (2008).

[18] R. Dreyfus, M. E. Leunissen, R. Sha, A. V. Tkachenko, N. C. Seeman, D. J. Pine, and P. M. Chaikin, Phys. Rev. Lett. 102, 048301 (2009).

[19] F. J. Martinez-Veracoechea, B. Bozorgui, and D. Frenkel, Soft Matter 6, 6136 (2010).

[20] M.E. Leunissen and D. Frenkel, J. Chem. Phys. 134, 084702 (2011).

[21] R. T. Scarlett, M. T. Ung, J. C. Crocker, and T. Sinno, Soft Matter 7, 1912 (2011).

[22] C. Knorowski, S. Burleigh, and A. Travesset, Phys. Rev. Lett. 106, 215501 (2011).

[23] W. B. Rogers and J.C. Crocker, Proc. Natl. Acad. Sci. U.S.A. 108, 15687 (2011).

[24] B. M. Mognetti, P. Varilly, S. Angioletti-Uberti, F. J. Martinez-Veracoechea, J. Dobnikar, M.E. Leunissen, and D. Frenkel, Proc. Natl. Acad. Sci. U.S.A. 109, E378 (2012).

[25] Y. Zhang, H. Zhou, and Z.-C. Ou-Yang, Biophys. J. 81, 1133 (2001)

[26] B. Tinland, A. Pluen, J. Sturm, and G. Weill, Macromolecules 30, 5763 (1997).

[27] S. Stranick, A. N. Parikh, D. L. Allara, and P. S. Weiss, J. Phys. Chem. 98, 11136 (1994).

[28] B. M. Mladek and D. Frenkel, Soft Matter 7, 1450 (2011).

[29] N. R. Markham and M. Zuker, Nucleic Acids Res. 33, W577 (2005).

[30] B. M. Mladek et al., "Multi-scale, ab initio coarse-graining of DNA-functionalized colloids" (to be published).

[31] Of course, the discrepancies between many-body interactions and two-body interactions will be configuration- and 
neighbor-dependent. While the chosen arrangement per se is not found in typical crystals, we chose it for convenient comparison with the data of $\Phi_{2}$. The mechanical stability of crystals is mediated by DNA bonds; thus, the chosen configuration is representative of crystalline arrangements in that several "like" next neighbors compete for strands of a given "unlike" colloid.
[32] D. Gottwald, G. Kahl, and C. N. Likos, J. Chem. Phys. 122, 204503 (2005).

[33] J. Fornleitner, F. Lo Verso, G. Kahl, and C. N. Likos, Soft Matter 4, 480 (2008).

[34] F. J. Martinez-Veracoechea, B. M. Mladek, A. V. Tkachenko, and D. Frenkel, Phys. Rev. Lett. 107, 045902 (2011). 